\title{
Energies and Alignment of Conjugated Molecules in the External Fields
}

\author{
Y. KABASHI AND S. KABASHI \\ University of Prishtina, Faculty of Mathematics and Natural Sciences, \\ Department of Physics, Prishtina, Kosovo
}

\begin{abstract}
This paper reports theoretical works based on experiments studying the formation and structure of thin films of a number of different organic molecules, particularly pentacene and a number of its functionalized variants and analogs on non-metallic substrates such as $\mathrm{KCl}$. The molecules that form the films studied are anisotropic planar molecules (i.e., they have the geometry, roughly, of domino tiles) of similar dimensions, but different charge distributions and free chemical bonds. In the films, these varying properties lead to different stacking schemes, including herringbone upright stacking, slip-stack stacking of flat-lying molecules, and cross-cross packing of molecules lying upright on the long edge. The energies of these planar organic molecules are studied in the presence of an electric field generated due to the surface charging. Then the energies of domino-shaped organic molecules are calculated in homogeneous and inhomogeneous electric fields, which model uniformly and non-uniformly charged substrates, respectively. The relevant properties of the molecules are given by the polarizability tensors and the quadrupole tensors of the respective molecules. These calculated elements of these two tensors are used to calculate the dipole and quadrupole contributions to their electrostatic energies in uniform electric fields and in non-uniform electric fields of point-charge form.
\end{abstract}

DOI: 10.12693/APhysPolA.137.458

PACS/topics: organic molecules-pentacene, homogeneous and inhomogeneous electric field, energies, alignment

\section{Introduction}

The formation and structure of thin films of a number of different organic molecules have special importance nowadays. Our work here is based on experiments carried out in the research group of Prof. Gregor Witte of the University of Marburg studying the formation and structure of thin films of a number of different organic molecules; in particular, of pentacene and a number of its functionalized variants and analogs on non-metallic substrates such as $\mathrm{KCl}$. The molecules that form the films studied are anisotropic planar molecules (i.e., have the geometry, roughly, of domino tiles) of similar dimensions, but different charge distributions and free chemical bonds [1]. In the films, these varying properties lead to different stacking schemes, including herringbone upright stacking, slip-stack stacking of flat-lying molecules, and cross-cross packing of molecules lying upright on the long edge (Fig. 1). In addition, the packing scheme depends on how the substrate is prepared. In particular, the stacking changes with an ionic bombardment of the substrate [1-5].

The leading experimentally based assumption for the effect of the bombardment of the surface is that the surface becomes charged [6-14]. We have studied the energies of these planar organic molecules in the presence of an electric field generated due to the surface charging. These energies are expected to have a decisive effect on the statics and dynamics of the film-formation process,

*corresponding author; e-mail: skender.kabashi@uni-pr.edu and understanding them is an essential first step for further understanding of the film formation $[1-4,15,16]$. In order to carry out this work, we have calculated the energies of domino-shaped organic molecules in homogeneous and inhomogeneous electric fields, which model uniformly and non-uniformly charged substrates, respectively.

The relevant properties of the molecules are given by the polarizability tensors and the quadrupole tensors of the respective molecules, which have recently been calculated using ab initio methods by Klues and Witte [1]. We have used these calculated elements of these two tensors to calculate the dipole and quadrupole contributions to their electrostatic energies in uniform and non-uniform electric fields of point-charge form.

\section{Calculation of energies and alignment of pentacene and its analogs}

Initially due to the fact that the surface (non-metallic surface $\mathrm{KCl}$ ) on which we place the molecule is negatively charged, it has been experimentally ascertained that if the surface is positively charged, it does not yield any results.

Now we will calculate the energies of domino-shaped organic molecules (Fig. 2) in homogeneous and inhomogeneous electric fields, which model, respectively, uniformly and non-uniformly charged substrates. For both cases in order to calculate the dipole and quadrupole contributions to the electrostatic energies, we used the relevant properties of the molecules. These are the polarizability tensors, as well as the quadrupole tensors of respective molecules. 
(a)

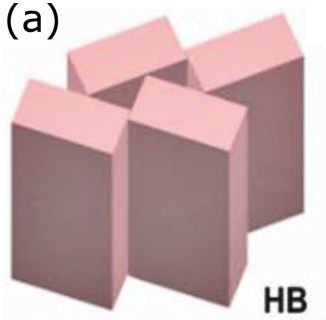

(c)

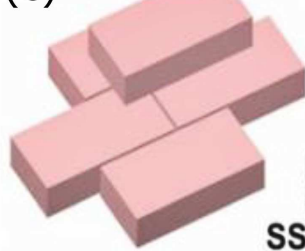

(e)
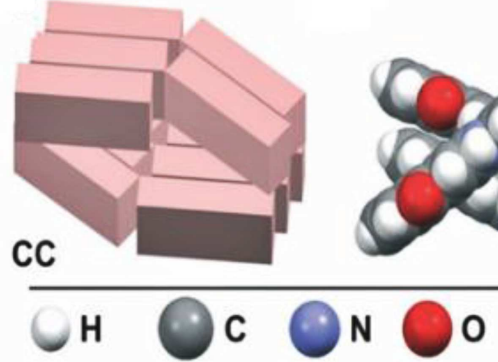

(f)

(b)

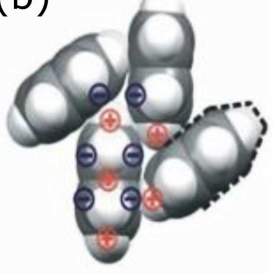

(d)
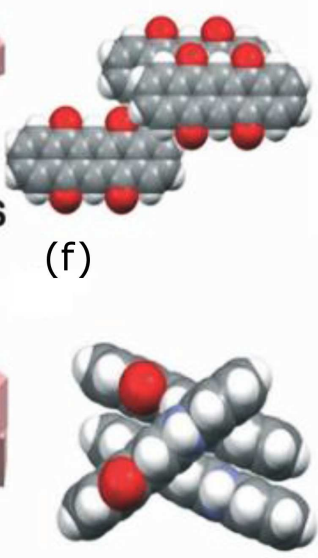
0

Fig. 1. Schematic depiction of the considered packing motifs (left column) and examples of their realization (right column): (a) herringbone (HB), (c) slip-stack (SS) and (e) cross-cross (CC) packing. HB packing is shown in (b) PEN together with charge distribution, indicating the molecular quadrupole moment, while part (d) reveals the SS-packing of TET, and (f) the CC arrangement of QUA- $\gamma$. The bottom row shows the color code that is used in the space-filling representations of the molecules [1].

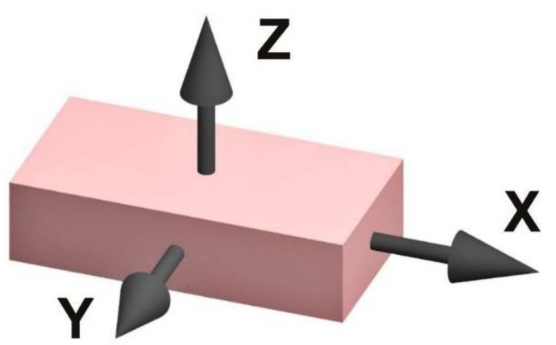

Fig. 2. General orientation of molecules within the coordinates system for calculation [1].

\subsection{Uniform electric field of point-charge form}

We first study the homogeneous electric field, which model uniformly charged substrates. This field will affect the polarizability of the molecule, and induce its dipole moment which then orients the molecule in the direction of field.

The quadrupole contribution, on the other hand, is present only when there is a local field gradient, i.e., when the field is sufficiently non-uniform. This means that in uniform field quadrupole contribution is zero, so the energy due to quadrupole moment is zero. This can be shown mathematically. We start from the relation obtained for multipole expansion of the energy of a charge distribution in an external field, where the quadrupole contribution is given by

$$
\begin{gathered}
U_{q}=-\frac{1}{6} \boldsymbol{\nabla} \cdot(\boldsymbol{Q} \cdot \boldsymbol{E})=-\frac{1}{6} \boldsymbol{\nabla} \cdot\left(\begin{array}{c}
Q_{x x} E_{x} \\
Q_{y y} E_{y} \\
Q_{z z} E_{z}
\end{array}\right)= \\
-\frac{1}{6}\left(Q_{x x} \frac{\partial E_{x}}{\partial x}+Q_{y y} \frac{\partial E_{y}}{\partial y}+Q_{z z} \frac{\partial E_{z}}{\partial z}\right)
\end{gathered}
$$

In the uniform field, the intensity components $E_{x}, E_{y}$, and $E_{z}$ are constants, so

$$
\frac{\partial E_{x}}{\partial x}=\frac{\partial E_{y}}{\partial y}=\frac{\partial E_{z}}{\partial z}=0
$$

Therefore, using this condition in (1), we obtain

$$
U_{q}=0 \text {. }
$$

It is clear now that in the uniform fields, only the dipole contribution is present.

To calculate energy and alignment we start with the expression for electrostatic energy of dipole [17, 18]:

$$
U=-\boldsymbol{p} \cdot \boldsymbol{E} .
$$

The polarizability tensor on the main axis of dipole is diagonal

$$
\alpha=\left(\begin{array}{ccc}
\alpha_{x x} & 0 & 0 \\
0 & \alpha_{y y} & 0 \\
0 & 0 & \alpha_{z z}
\end{array}\right)
$$

while the electric field vector is

$$
\boldsymbol{E}=\left(E_{x} \boldsymbol{e}_{x}+E_{y} \boldsymbol{e}_{y}+E_{z} \boldsymbol{e}_{z}\right)=\left(\begin{array}{c}
E_{x} \\
E_{y} \\
E_{z}
\end{array}\right)
$$

In this case for the field direction we get three components of polarizability

$$
\begin{gathered}
\boldsymbol{p}=\alpha \cdot \boldsymbol{E}=\left(\begin{array}{ccc}
\alpha_{x x} & 0 & 0 \\
0 & \alpha_{y y} & 0 \\
0 & 0 & \alpha_{z z}
\end{array}\right) \cdot\left(\begin{array}{c}
E_{x} \\
E_{y} \\
E_{z}
\end{array}\right)= \\
\left(\begin{array}{c}
\alpha_{x x} E_{x} \\
\alpha_{y y} E_{y} \\
\alpha_{z z} E_{z}
\end{array}\right) \equiv \alpha_{x x} E_{x} \boldsymbol{e}_{x}+\alpha_{y y} E_{y} \boldsymbol{e}_{y}+\alpha_{z z} E_{z} \boldsymbol{e}_{z} .
\end{gathered}
$$

Thus,

$$
\boldsymbol{p}=\left(\begin{array}{c}
p_{x} \\
p_{y} \\
p_{z}
\end{array}\right)=\left(\begin{array}{c}
\alpha_{x x} E_{x} \\
\alpha_{y y} E_{y} \\
\alpha_{z z} E_{z}
\end{array}\right)
$$




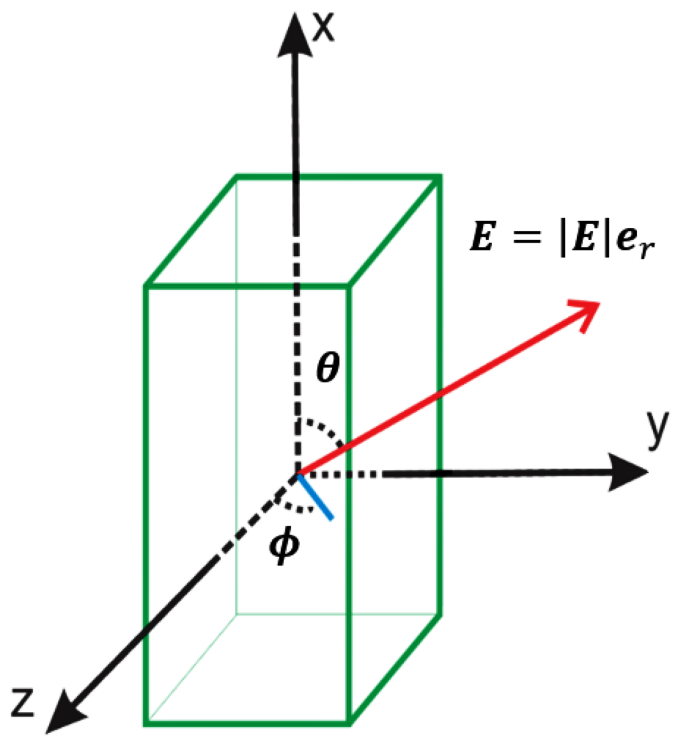

Fig. 3. General orientation of molecules within the coordinates system for calculation in spherical coordinates.

Then the electrostatic energy of the dipole in the Cartesian coordinate system is

$$
\begin{gathered}
U_{d}=-\boldsymbol{p} \cdot \boldsymbol{E}=-\left(\begin{array}{c}
\alpha_{x x} E_{x} \\
\alpha_{y y} E_{y} \\
\alpha_{z z} E_{z}
\end{array}\right) \cdot\left(\begin{array}{c}
E_{x} \\
E_{y} \\
E_{z}
\end{array}\right)= \\
-\left(\alpha_{x x} E_{x}^{2}+\alpha_{y y} E_{y}^{2}+\alpha_{z z} E_{z}^{2}\right)
\end{gathered}
$$

Now we want to describe the direction of molecule. To describe an arbitrary direction, probably the easiest thing to do is to use the directions given in spherical coordinates (Fig. 3).

The electrostatic field vector in spherical coordinates is given by

$$
\boldsymbol{E}=|\boldsymbol{E}| \boldsymbol{e}_{r},
$$
where $\boldsymbol{e}_{r}=\sin (\theta) \cos (\phi) \boldsymbol{e}_{x}+\sin (\theta) \sin (\phi) \boldsymbol{e}_{y}+\cos (\theta) \boldsymbol{e}_{z}$.

Thus, for the electrostatic field vector we obtain

$$
\boldsymbol{E}=|\boldsymbol{E}|\left(\begin{array}{c}
\sin (\theta) \cos (\phi) \\
\sin (\theta) \sin (\phi) \\
\cos (\theta)
\end{array}\right) \text {. }
$$

Then the dipole moment is

$$
\begin{aligned}
& \qquad \begin{array}{l}
\boldsymbol{p}=\alpha \boldsymbol{E}=|\boldsymbol{E}|\left(\begin{array}{c}
\alpha_{x x} \sin (\theta) \cos (\phi) \\
\alpha_{y y} \sin (\theta) \sin (\phi) \\
\alpha_{z z} \cos (\theta)
\end{array}\right) \\
\boldsymbol{p}=|\boldsymbol{E}| \alpha_{x x} \sin (\theta) \cos (\phi) \boldsymbol{e}_{x}+|\boldsymbol{E}| \alpha_{y y} \sin (\theta) \sin (\phi) \boldsymbol{e}_{y} \\
\quad+|\boldsymbol{E}| \alpha_{z z} \cos (\theta) \boldsymbol{e}_{z},
\end{array} \\
& \text { while the electrostatic energy of the dipole is } \\
& \quad U_{d}=-\boldsymbol{p} \cdot \boldsymbol{E}
\end{aligned}
$$

After substituting the expression for dipolar moment we get

$$
\begin{aligned}
U_{d} & =-|\boldsymbol{E}|^{2}\left(\alpha_{x x} \sin ^{2}(\theta) \cos ^{2}(\phi)\right. \\
& \left.+\alpha_{y y} \sin ^{2}(\theta) \sin ^{2}(\phi)+\alpha_{z z} \cos ^{2}(\theta)\right)
\end{aligned}
$$

Since the intensity of electric field of a point charge is $|\boldsymbol{E}|=\frac{1}{4 \pi \varepsilon_{0}} \frac{q}{r^{2}}=\frac{k q}{r^{2}}$, and $|\boldsymbol{E}|^{2}=\left(k \frac{q}{r^{2}}\right)^{2}=\frac{k^{2} q^{2}}{r^{4}}$, therefore

$$
\begin{aligned}
U_{d} & =-\frac{k^{2} q^{2}}{r^{4}}\left(\sin ^{2}(\theta)\left(\alpha_{x x} \cos ^{2}(\phi)+\alpha_{y y} \sin ^{2}(\phi)\right)\right. \\
& \left.+\alpha_{z z} \cos ^{2}(\theta)\right),
\end{aligned}
$$

where $0 \leq \theta \leq \pi, 0 \leq \phi \leq 2 \pi, k$ is electrostatic (Coulomb constant) $\left[\mathrm{N} \mathrm{m}^{2} / \mathrm{C}^{2}\right], q$ is electric charge of the electron $[\mathrm{C}], r$ is a distance between ions in $\mathrm{KCl}$ molecule [m], and $\alpha_{x x}, \alpha_{y y}$ and $\alpha_{z z}$ are polarizability components, $\alpha_{z z}<\alpha_{y y}<\alpha_{x x}\left[\mathrm{~m}^{3}\right]$.

We found that the distance-dependence of the dipole contribution goes inversely as the fourth power of distance. This is because the dipole moment itself must be induced by the field.

\subsubsection{Results for uniform electric field of point-charge form}

MATLAB(R2018a) [19] is used to map orientationdependence of the electrostatic energies in uniform electric field, using (16).

From the experiment we know the value of $r=1.57 \times 10^{-10} \mathrm{~m}$ and the corresponding polarizability components $\left(\alpha_{x x}, \alpha_{y y}\right.$, and $\left.\alpha_{z z}\right)$ for the respective molecules (Table I).

In Fig. 4 we show the maps of the orientationdependence of the electrostatic energies contribution for all nine pentacene analogs in the uniform electric field of point-charge form. In all other patterns we see that the points where we have maximum and minimum energy for all pentacene analogs that we analyzed do not change, and only the depths of the patterns change.

From maps of the orientation-dependence we see that for $\theta=0$ and $\theta=\pi$ we have maximum energy (since it has negative sign, for the experimentalists is known as minimum energy).

TABLE I

Computed $\alpha$-polarizability of all molecules in $10^{-24} \mathrm{~cm}^{3}=10^{-30} \mathrm{~m}^{3}$. Static dipole polarizability was calculated as $\alpha_{t o t}=\frac{1}{3}\left(\alpha_{x x}+\alpha_{y y}+\alpha_{z z}\right)$ [1].

\begin{tabular}{l|c|c|c|c}
\hline \hline & $\alpha_{x x}$ & $\alpha_{y y}$ & $\alpha_{z z}$ & $\alpha_{t o t}$ \\
\hline PEN & 90.7 & 36.2 & 12.9 & 46.6 \\
PFP & 94.8 & 38.1 & 13.0 & 48.7 \\
HFDP & 89.5 & 34.5 & 12.7 & 45.6 \\
DAP & 89.4 & 33.9 & 12.6 & 45.3 \\
DHDAP & 85.1 & 33.2 & 12.9 & 43.7 \\
QUI & 77.2 & 36.2 & 12.9 & 42.1 \\
TET & 65.2 & 39.1 & 13.0 & 39.1 \\
QUA (diag.) & 69.9 & 36.9 & 13.0 & 40.0 \\
DNTT (diag.) & 93.5 & 40.0 & 14.4 & 49.3
\end{tabular}



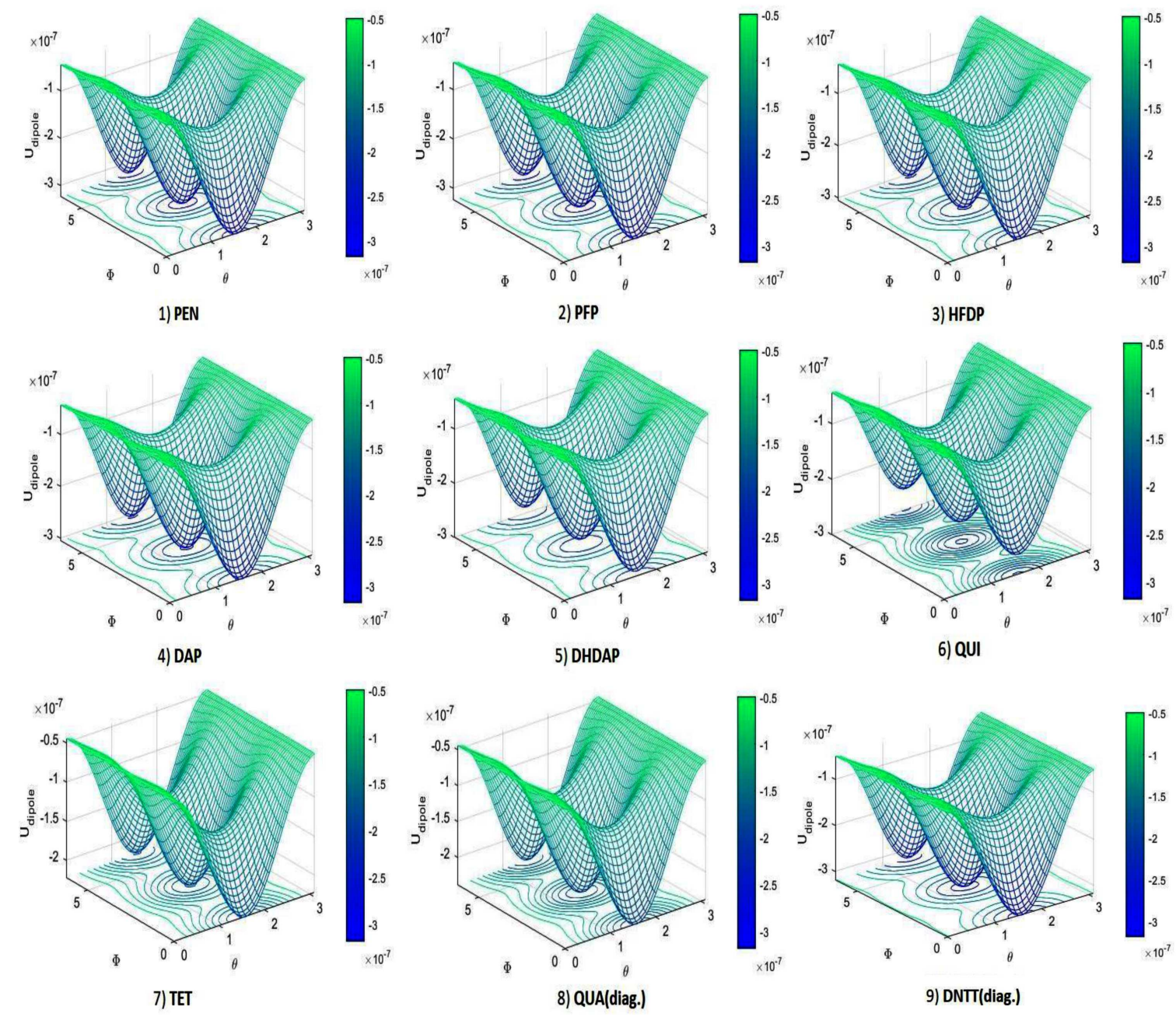

Fig. 4. Maps of the orientation-dependence of the electrostatic energies for all nine pentacene analogs in the uniform electric field of point-charge form.

The energies of all molecules cause the axis of maximum polarizability, which in all cases is the long axis of the molecule, to align with the field.

\subsection{Non-uniform electric field of point-charge form}

A non-uniform field affects the molecular polarizability and besides dipole moment (which was the only contribution for the uniform field), now we will also have the quadrupolar moment.

Therefore, the total energy of the molecule is the sum of these two contributions, i.e., the contribution of the dipolar moment and the contribution of the quadrupolar moment.

Since the dipole contribution depends largely on the local vector, the result we obtained in the case of the uniform field also applies to the case of a non-uniform field. So, we only have to find the contribution of the quadrupolar moment, and then collect them.

We treated those two contributions separately just to analyze better and to understand the effects.
So, now we will find the energy due to the quadrupolar moment. To find this we start with the expression for electrostatic energy in multipoles [17]:

$$
W=q \Phi-\boldsymbol{p} \cdot \boldsymbol{E}-\frac{1}{6} \boldsymbol{\nabla} \cdot(\boldsymbol{Q} \cdot \boldsymbol{E})+\ldots
$$

where the third term in this equation represents the energy due to the quadrupole moment. So, energy due to the quadrupolar moment is obtained by

$$
U_{q}=-\frac{1}{6} \nabla \cdot(\boldsymbol{Q} \cdot \boldsymbol{E})
$$

If we work in the proper coordinate system for the molecule and imagine the molecule as domino-shaped we will have the case as in Fig. 2, and we get

$$
U_{q}=\frac{1}{6}\left(Q_{x x} \frac{\partial E_{x}}{\partial x}+Q_{y y} \frac{\partial E_{y}}{\partial y}+Q_{z z} \frac{\partial E_{z}}{\partial z}\right) .
$$

Since for us, quadrupole components $\left(Q_{x x}, Q_{y y}\right.$, and $\left.Q_{z z}\right)$ and also the electric field intensity are knownquantities, but the components of the electric field $\left(E_{x}\right.$, $E_{y}$, and $E_{z}$ ) are unknown, we make some transformations of these three components. 
We will start with the electric field vector given by

$$
\boldsymbol{E}(x, y, z)=|\boldsymbol{E}| \hat{r} \text {. }
$$

We know that the intensity of the electric field of point charge form is given by $|\boldsymbol{E}|=\frac{1}{4 \pi \varepsilon_{0}} \frac{q}{r^{2}}$.

So, for the electric field vector we get

$$
\begin{aligned}
& \boldsymbol{E}(x, y, z)=E_{x} \boldsymbol{e}_{x}+E_{y} \boldsymbol{e}_{y}+E_{z} \boldsymbol{e}_{z} \\
& E_{x}=k q \frac{x}{\left(x^{2}+y^{2}+z^{2}\right)^{\frac{3}{2}}}, \quad E_{y}=k q \frac{y}{\left(x^{2}+y^{2}+z^{2}\right)^{\frac{3}{2}}}, \\
& E_{z}=k q \frac{z}{\left(x^{2}+y^{2}+z^{2}\right)^{\frac{3}{2}}} .
\end{aligned}
$$

Now we need to find the partial derivative of the relevant components of the electric field. After finding $\frac{\partial E_{x}}{\partial x} \frac{\partial E_{y}}{\partial y}$ and $\frac{\partial E_{z}}{\partial z}$ and substituting those three expressions in (19) we get

$$
\begin{aligned}
U_{q} & =-\frac{k q}{6 r^{5}}\left[Q_{x x}\left(-2 x^{2}+y^{2}+z^{2}\right)\right. \\
& \left.+Q_{y y}\left(x^{2}-2 y^{2}+z^{2}\right)+Q_{z z}\left(x^{2}+y^{2}-2 z^{2}\right)\right] .
\end{aligned}
$$

After we transform the coordinates from Cartesian to spherical coordinates

$$
\left.\begin{array}{l}
x=r \sin (\theta) \cos (\phi) \\
y=r \sin (\theta) \sin (\phi) \\
z=r \cos (\theta)
\end{array}\right\} \sim\left\{\begin{array}{l}
x^{2}=r^{2} \sin ^{2}(\theta) \cos ^{2}(\phi) \\
y^{2}=r^{2} \sin ^{2}(\theta) \sin ^{2}(\phi) \\
z^{2}=r^{2} \cos ^{2}(\theta)
\end{array}\right.
$$

the final expression for the quadrupole electrostatic energy is

$$
\begin{aligned}
U_{q} & =-\frac{k q}{6 r^{3}}\left[Q_{x x}\left(1-3 \sin ^{2}(\theta) \cos ^{2}(\phi)\right)\right. \\
& \left.+Q_{y y}\left(1-3 \sin ^{2}(\theta) \sin ^{2}(\phi)\right)+\left(1-3 \cos ^{2}(\theta)\right)\right],
\end{aligned}
$$

where $Q_{x x}, Q_{y y}$, and $Q_{z z}$ are quadrupole moments.

We found that the distance-dependence of the quadrupole contribution goes inversely to the third power of distance. This is because the quadrupole moment is static. Consequently, the quadrupolar contribution at larger distances becomes generally more significant.

Therefore, the total energy of the molecule in the nonuniform field will be the sum of these two contributions, i.e., the contribution of the dipole moment and the contribution of the quadrupole moment

$$
\begin{aligned}
U & =U_{\text {tot }}=U_{d}+U_{q} \\
U & =-\frac{k^{2} q^{2}}{r^{4}}\left(\alpha_{x x} \sin ^{2}(\theta) \cos ^{2}(\phi)\right. \\
& \left.+\alpha_{y y} \sin ^{2}(\theta) \sin ^{2}(\phi)+\alpha_{z z} \cos ^{2}(\theta)\right) \\
& -\frac{k q}{6 r^{3}}\left[Q_{x x}\left(1-3 \sin ^{2}(\theta) \cos ^{2}(\phi)\right)\right. \\
& \left.+Q_{y y}\left(1-3 \sin ^{2}(\theta) \sin ^{2}(\phi)\right)+Q_{z z}\left(1-3 \cos ^{2}(\theta)\right)\right] .
\end{aligned}
$$

2.2.1. Results for non-uniform electric field of pointcharge form

To have a better understanding of the quadrupole contribution we plotted the orientation-dependence of the electrostatic energies only from the quadrupole contribution (25) for all nine pentacene analogs using MATLAB(R2018a) [19].

From the experiment we know the value of $r=1.57 \times 10^{-10} \mathrm{~m}$, and the corresponding quadrupole moments $\left(Q_{x x}, Q_{y y}\right.$, and $\left.Q_{z z}\right)$ for the respective molecules (Table II).

In Fig. 5 we see the maps of the orientation-dependence of the electrostatic energies only from quadrupole contribution for all nine pentacene analogs in the non-uniform electric field.

From the obtained models we see that each molecule we treated has different pattern. This implies that the quadrupolar tensor elements vary significantly from molecule to molecule, so the orientation of the molecule leading to the minimum energy is different for the different molecules and is generally not same as the dipolar contribution.

Thus, in the non-uniform field, the total energy of the molecule is the sum of two contributions: the dipole moment and the quadrupole moment.(26) (see Fig. 6).

In Fig. 6 we have carefully calculated these energies for all nine pentacene analogs [1], made maps of the orientation-dependence of the electrostatic energies taking into account the different distance-dependences of the competing interactions.

From obtained results we see that in the inhomogeneous electric field for small distances, the dipole moment dominates. We can see this when we look at the obtained patterns which show similarity to the case treated in homogeneous field when we only had dipole contribution. The consequence is that the quadrupolar contribution is actually more important at larger distances.

\section{TABLE II}

Calculated quadrupole moments of all molecules in $10^{-34} \mathrm{C} \mathrm{cm}^{2}=10^{-38} \mathrm{C} \mathrm{m}^{2}$. Magnitude of quadrupole moments was given by $Q_{\mathrm{tot}}=\sqrt{\frac{2}{3}\left(Q_{x x}^{2}+Q_{y y}^{2}+Q_{z z}^{2}\right)}[1]$.

\begin{tabular}{l|c|c|c|c}
\hline \hline & $Q_{x x}$ & $Q_{y y}$ & $Q_{z z}$ & $Q_{t o t}$ \\
\hline PEN & 4.9 & 4.0 & -8.9 & 8.9 \\
PFP & -3.7 & -5.5 & 9.2 & 9.2 \\
HFDP & 9.4 & -6.8 & -2.6 & 9.7 \\
DAP & 10.7 & -1.7 & -9.0 & 11.5 \\
DHDAP & -2.5 & 9.0 & -6.5 & 9.3 \\
QUI & 16.3 & -7.6 & -8.8 & 16.3 \\
TET & 21.1 & -15.3 & -5.8 & 21.8 \\
QUA (diag.) & 17.7 & -11.3 & -6.4 & 17.9 \\
DNTT (diag.) & -10.0 & 1.9 & 8.1 & 10.7
\end{tabular}



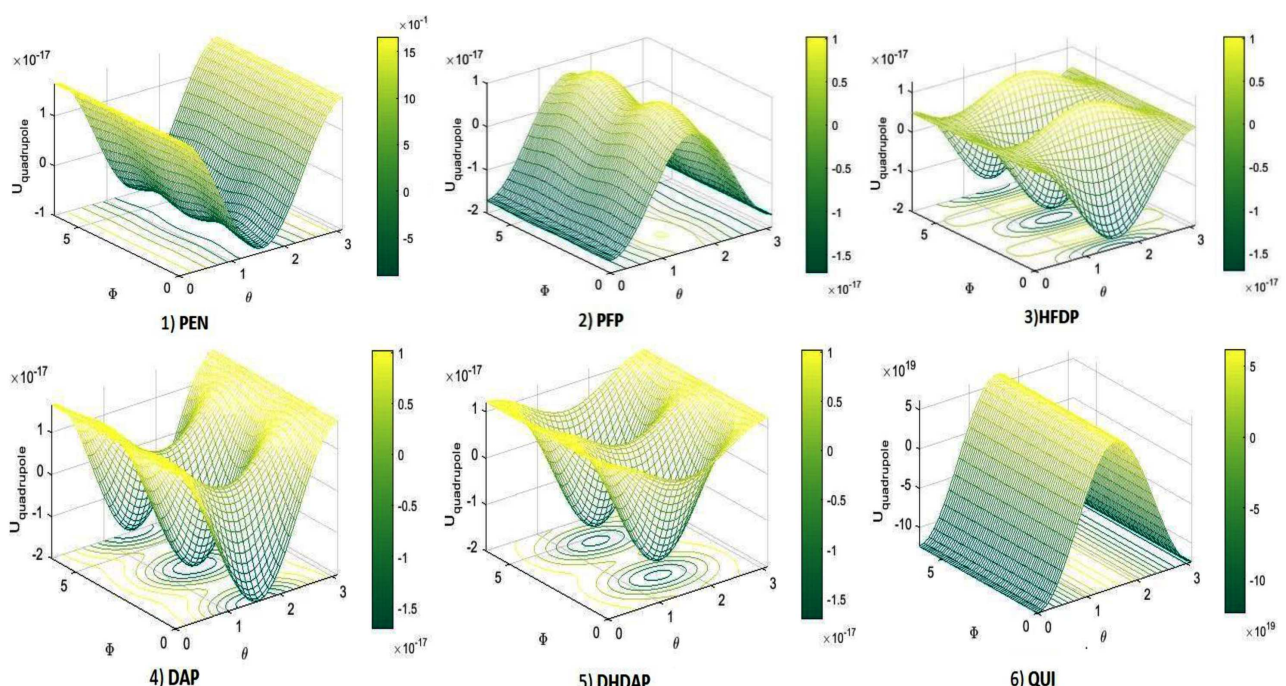

5) DHDAP

6) QUI

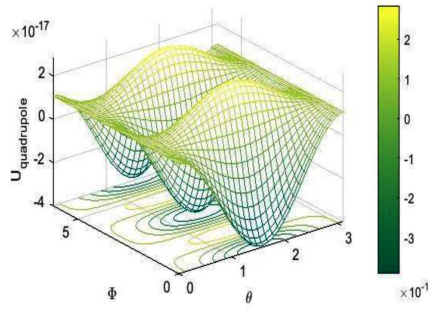

7) TET
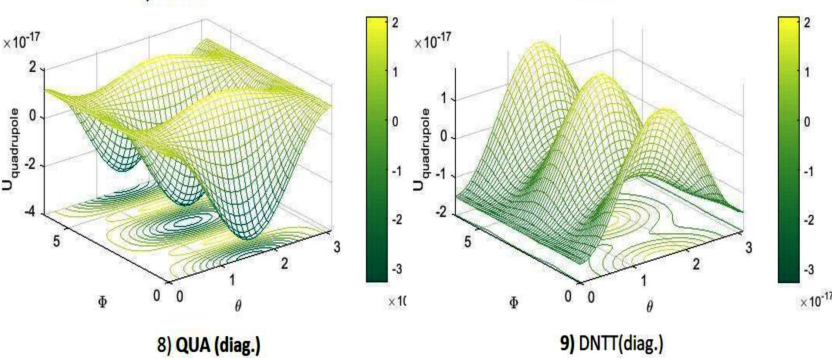

Fig. 5. Maps of the orientation-dependence of the electrostatic energies only from quadrupole contribution for all nine pentacene analogs in the non-uniform electric field of point-charge form.
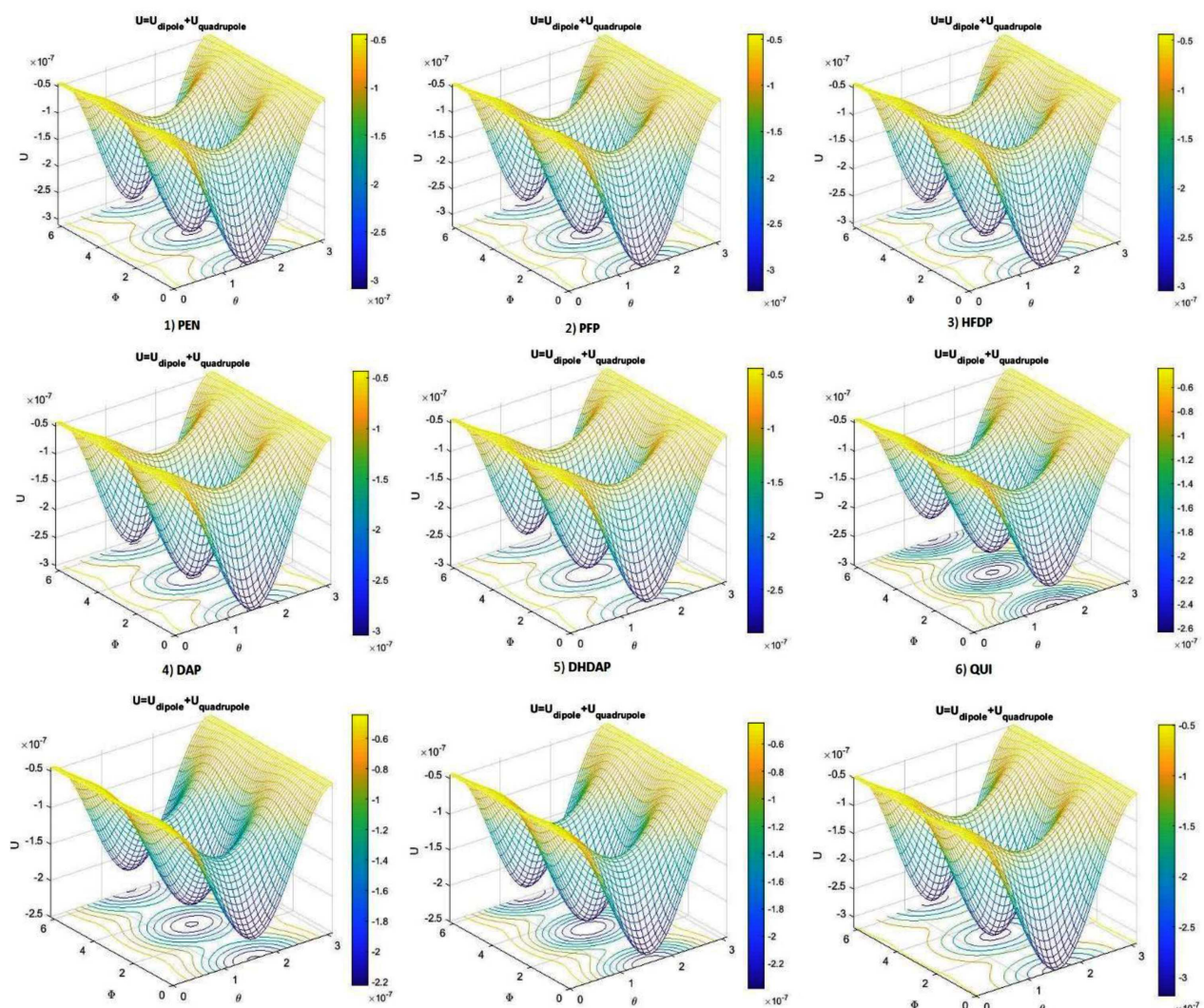

7) TET

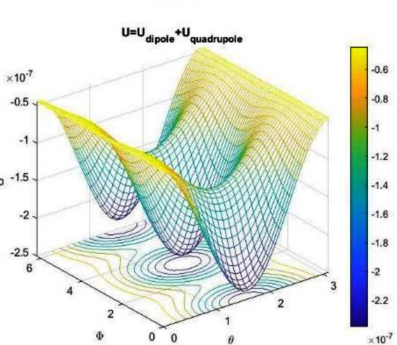

8) QUA (diag.)

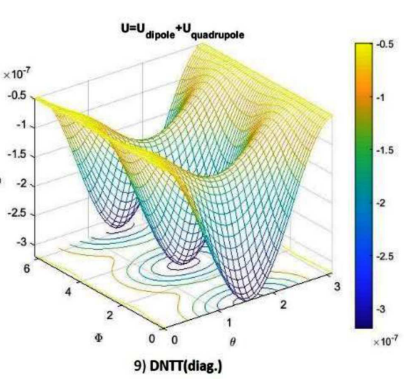

Fig. 6. Maps of the orientation-dependence of the electrostatic energies for all nine pentacene analogs in the nonuniform electric field of of point-charge form.. 


\section{Conclusion}

The energies of the preferred orientation of the molecules can be determined by the competition between the dipole and the quadrupole interactions, with their relative strengths also depending on the distance from the charge center.

We have found two interesting results for the case of a non-uniform Coulomb field:

1. the distance-dependence of the dipole contribution goes inversely as the fourth power of distance vs. the third power for the quadrupole contribution. This is because the dipole moment itself must be induced by the field, whereas the quadrupole moment is static. The consequence is that the quadrupolar contribution is actually more important at larger distances.

2. The elements of the quadrupole tensor vary significantly from molecule to molecule, so that orientation of the molecule that leads to minimum energy is different for different molecules and, in general, is not the same as the dipole contribution.

This work makes a necessary and useful contribution to understanding specific experimental results for the stacking patterns of thin films of pentacene-like organic molecules.

\section{Acknowledgments}

The authors express their gratitude to Prof. Reinhard M. Noack and Prof. Gregor Witte for contributions and for providing the relevant properties and dates of the molecules treated in this paper.

\section{References}

[1] M. Klues, G. Witte, Phys. Chem. CrystEngComm 20, 63 (2018).

[2] R. Felix, T. Breuer, G. Witte, K. Volz, K.I. Gries, J. Cryst. Growth 471, 29 (2017).
[3] T. Breuer, A. Karthäuser, H.W. Klemm, F. Genuzio, G. Peschel, A. Fuhrich, T. Schmidt, G. Witte, ACS Appl. Mater. Interfaces 9, 8384 (2017).

[4] A. Mänz, A. Aline Hauke, G. Witte, J. Phys. Chem. C 122, 2165 (2018).

[5] C. Cocchi, T. Breuer, G. Witte, C. Draxl, Phys. Chem. Chem. Phys. 20, 29724 (2018).

[6] H. Md Faruk, P. Md Sarwar, N. Mai, Emerging Mater. Res. 9, 1 (2020).

[7] H. Soni, M.P. Madindwa, Emerging Mater. Res. 9, 1 (2020).

[8] O. Neslihan, O. Murat, Emerging Mater. Res. 9, 1 (2020).

[9] Y. Zafer, A. Lutfiye, S. Mehmet, Emerging Mater. Res. 9, 1 (2020).

[10] Y. Ayça Kıyak, A. Barış, Emerging Mater. Res. 9, 1 (2020).

[11] Li Xiaojie, Zhao Kai, Emerging Mater. Res. 8, 77 (2019).

[12] M. Amit, S. Sarabjeet Singh, D. Sandeep, Emerging Mater. Res. 9, 1 (2020).

[13] N. Özsoy, Int. J. Computat. Exp. Sci. Eng. (IJCESEN) 5, 105 (2019).

[14] R.U. Mullai, R. Jothi Arul, T. Suresh, E. Vinoth, S. Gopinath, G. Vinitha, K. Rao Sreenadha, S. Vetrivel, Emerging Mater. Res. 9, 1 (2020).

[15] L. von Helden, T. Breuer, G. Witte, Appl. Phys. Lett. 110, 141904 (2017).

[16] M. Dreher, D. Bischof, F. Widdascheck, A. Huttner, T. Breuer, G. Witte, Adv. Mater. Interfaces $\mathbf{5}$, 1800920 (2018).

[17] J.D. Jackson, Classical Electrodynamics, Wiley, New York 1999

[18] D. Griffiths, Introduction to Electrodynamics, Cambridge University Press, Cambridge 2017.

[19] MATLAB 2018a, The MathWorks, Inc., Natick (MA). 